\title{
Correction to: Status and trends of circumpolar peregrine falcon and gyrfalcon populations
}

\author{
Alastair Franke, Knud Falk (1), Kevin Hawkshaw, Skip Ambrose, \\ David L. Anderson, Peter J. Bente, Travis Booms, Kurt K. Burnham, \\ Suzanne Carrière, Johan Ekenstedt, Ivan Fufachev, \\ Sergey Ganusevich, Kenneth Johansen, Jeff A. Johnson, \\ Sergey Kharitonov, Pertti Koskimies, Olga Kulikova, \\ Peter Lindberg, Berth-Ove Lindström, William G. Mattox, \\ Carol L. McIntyre, Svetlana Mechnikova, Dave Mossop, \\ Søren Møller, Ólafur K. Nielsen, Tuomo Ollila, Arve Østlyngen, \\ Ivan Pokrovsky, Kim Poole, Marco Restani, Bryce W. Robinson, \\ Robert Rosenfield, Aleksandr Sokolov, Vasiliy Sokolov, \\ Ted Swem, Katrin Vorkamp
}

Published online: 21 January 2020

Correction to: Ambio

https://doi.org/10.1007/s13280-019-01300-z

While collating contributions and comments from 36 researchers, the coordinating authors accidentally omitted Dr. Suzanne Carrière from the list of contributing co-authors. Dr. Carrière's data are described in Tables 1 and 3, Figure 2 and several places in the narrative.

The new author list is thus updated in this article.

Publisher's Note Springer Nature remains neutral with regard to jurisdictional claims in published maps and institutional affiliations.

\section{AUTHOR BIOGRAPHY}

\author{
Alastair Franke \\ Address: Department of Biological Sciences, University of Alberta, \\ CW 405, Biological Sciences Bldg., Edmonton, AB T6G 2E9, \\ Canada. \\ Address: Arctic Raptor Project, P.O. Box 626, Rankin Inlet, NT X0C \\ 0G0, Canada. \\ e-mail: alastair.franke@ulaberta.ca
}

\section{Knud Falk ( $\square)$}

Address: Stockholm, Sweden

e-mail: knudfalk@hotmail.com

https://www.vandrefalk.dk

\section{Kevin Hawkshaw}

Address: Department of Renewable Resources, University of Alberta, Edmonton, Canada.

e-mail: hawkshaw@ualberta.ca

The original article can be found online at https://doi.org/10.1007/ s13280-019-01300-z.
Skip Ambrose

Address: Fairbanks, USA.

e-mail: skipambrose@frontiernet.net

David L. Anderson

Address: The Peregrine Fund, 5668 W. Flying Hawk Lane, Boise, ID 83709, USA.

e-mail: danderson@peregrinefund.org

Peter J. Bente

Address: Alaska Department of Fish \& Game, Juneau, USA.

e-mail: pjbente@cox.net

Travis Booms

Address: Alaska Department of Fish \& Game, Juneau, USA.

e-mail: travis.booms@alaska.gov

\section{Kurt K. Burnham}

Address: High Arctic Institute, 603 10th Avenue, Orion, IL 61273, USA.

e-mail: kburnham@higharctic.org

Suzanne Carrière has served as Wildlife Biologist at Government of the Northwest Territories for 20 years and provides overall direction on biodiversity programs. Her expertise covers species status assessments and long-term monitoring of northern ecosystems and she serves as a member on the Committee on the Status of Endangered Wildlife in Canada (COSEWIC). She also serves on a comanagement board and other working groups on biodiversity and status of wildlife in Canada.

Address: Environment and Natural Resources, Government of the Northwest Territories, Yellowknife, NT X1A 2L9, Canada. e-mail: Suzanne_Carriere@gov.nt.ca

Johan Ekenstedt

Address: Tavelsjö, Sweden.

e-mail: jekenstedt@gmail.com 


\section{Ivan Fufachev}

Address: Arctic Research Station of Institute of Plant and Animal Ecology Ural Branch, Russian Academy of Sciences, Zelenaya Gorka Str., 21, Labytnangi, Yamal-Nenets Autonomous District, Russia 629400 .

e-mail: fufa4ew@yandex.ru

\section{Sergey Ganusevich}

Address: Center for Rescue of Wild Animals (Independent Non-profit Organization), Moscow, Russia.

e-mail: sganusevich@mail.ru

\section{Kenneth Johansen}

Address: Raptor Group Finnmark, Alta, Norway.

e-mail: kennethalta@hotmail.com

\section{Jeff A. Johnson}

Address: Department of Biological Sciences, Advanced Environmental Research Institute, University of North Texas, 1155 Union Circle, \#310559, Denton, TX 76203, USA.

e-mail: jeff.johnson@unt.edu

\section{Sergey Kharitonov}

Address: Bird Ringing Centre of Russia IEE RAS, Moscow, Russia 117312.

e-mail: serpkh@gmail.com

\section{Pertti Koskimies}

Address: Kirkkonummi, Finland.

e-mail: pertti.koskimies@kolumbus.fi

\section{Olga Kulikova}

Address: Institute of Biological Problems of the North, 18 Portovaya Street, Magadan, Russia 685000.

e-mail: gaerlach@gmail.com

\section{Peter Lindberg}

Address: Department of Biological and Environmental Sciences, University of Gothenburg, Box 463, 40530 Göteborg, Sweden.

e-mail: peter.lindberg@bioenv.gu.se

\section{Berth-Ove Lindström}

Address: Boden, Sweden.

e-mail: berthove.lindstrom@gmail.com

\section{William G. Mattox}

Address: Conservation Research Foundation, 702 S. Spelman Ln, Meridian, ID, USA.

e-mail:wgmattox2@earthlink.net

\section{Carol L. McIntyre}

Address: US National Park Service, Alaska, USA.

e-mail: carol_mcintyre@nps.gov

\section{Svetlana Mechnikova}

Address: I. M. Sechenov First Moscow State Medical University (Sechenov University), 119991 Trubetskaya 8, Moscow, Russia. e-mail: mechnikova@yandex.ru

\section{Dave Mossop}

Address: Yukon Research Centre, Whitehorse, Canada.

e-mail: dmossop@yukoncollege.yk.ca

\section{Søren Møller}

Address: Roskilde University, P.O. Box 260, 4000 Roskilde, Denmark.

e-mail: moller@ruc.dk

https://www.vandrefalk.dk

\section{Ólafur K. Nielsen}

Address: Icelandic Institute of Natural History, Garðabær, Iceland. e-mail: okn@ni.is

Tuomo Ollila

Address: Metsähallitus, Parks and Wildlife Finland, Rovaniemi, Finland.

e-mail: tuomo.ollila@metsa.fi

\section{Arve Østlyngen}

Address: Raptor Group Finnmark, Alta, Norway.

e-mail: aoestly@online.no

\section{Ivan Pokrovsky}

Address: Department of Migration, Max Planck Institute of Animal Behavior, Am Obstberg 1, 78315 Radolfzell, Germany.

Address: Laboratory of Ornithology, Institute of Biological Problems of the North FEB RAS, 18 Portovaya Str., Magadan, Russia 685000. Address: Arctic Research Station, Institute of Plant \& Animal Ecology, UD RAS, 21 Zelyonaya Gorka, Labytnangi, Russia 629400. e-mail: ivanpok@mail.ru

\section{Kim Poole}

Address: Aurora Wildlife Research, Nelson, Canada. e-mail: kpoole@aurorawildlife.com

\section{Marco Restani}

Address: St. Cloud State University, St. Cloud, USA. e-mail: Restani@stcloudstate.edu

\section{Bryce W. Robinson}

Address: The Peregrine Fund, and Ornithologi, Boise, USA.

e-mail: bryce@ornithologi.com

\section{Robert Rosenfield}

Address: University of Wisconsin - Stevens Point, Stevens Point, USA.

e-mail: rrosenfi@uwsp.edu

\section{Aleksandr Sokolov}

Address: Arctic Research Station of Institute of Plant and Animal Ecology Ural Branch, Russian Academy of Sciences, Zelenaya Gorka Str., 21, Labytnangi, Yamal-Nenets Autonomous District, Russia 629400.

e-mail:sokhol@yandex.ru

\section{Vasiliy Sokolov}

Address: Institute of Plant and Animal Ecology Ural Branch, Russian Academy of Sciences, Ekaterinburg, Russia.

e-mail: vsokolov@inbox.ru

\section{Ted Swem}

Address: U.S. Fish and Wildlife Service, Alaska, USA.

e-mail: ted_swem@fws.gov

\section{Katrin Vorkamp}

Address: Department of Environmental Science, Aarhus University, Roskilde, Denmark.

e-mail: kvo@envs.au.dk 